\title{
« Relatif neutre » et progression narrative : étude de cas dans un récit préclassique
}

\author{
Annie Kuyumcuyan ${ }^{1, *}$ \\ ${ }^{1}$ Université de Strasbourg - LiLPa
}

Résumé. Le relatif neutre ce qui/-e existe depuis l'ancien français mais s'est vu adjoindre le démonstratif neutre à partir du moyen français, les deux formes coexistant jusqu'à l'époque classique. Le but de cet article est de dénombrer et comparer les formes de relatif neutre (avec et sans démonstratif) dans un récit du $16^{\mathrm{e}}$ siècle, L'Heptameron, pour (i) déterminer si les deux formes apparaissent dans les mêmes contextes (ii) étudier leur rôle dans les enchaînements textuels. Étant donné leur nombre important, on ne peut que s'étonner de leur disparition relativement subite au siècle suivant, non moins que de leur réapparition progressive en français moderne; disparition et réapparition à l'observation desquelles cette étude voudrait contribuer.

\begin{abstract}
Neutral relative pronoun and narrative progression: case study in a pre-classical narrative. The relative neutral has been existing since Old French but was added with the demonstrative neutral from Middle French on, both forms coexisting until the classical period. The purpose of this article is to count and compare the forms of relative neutral (with and without demonstrative) in a 16th century narrative, L'Heptameron, in order to (i) determine whether the two forms appear in the same contexts, and (ii) study their role in textual sequences. Given their large number, one cannot but be astonished by their relatively sudden disappearance in the following century, no less than by their gradual reappearance in modern French; disappearance and reappearance to which this study would like to contribute.
\end{abstract}

\section{Un « relatif neutre » anaphorique}

\subsection{En français moderne}

Le français moderne présente un emploi «anaphorique» de la séquence démonstratif neutre $c e+$ relatif, à ne pas confondre avec la séquence homonyme des relatives dites «périphrastiques» (GMF 2009: 814-815). Si ce peut alors toujours être tenu pour l'«antécédent» du relatif, il n'y est pas, comme dans les structures concurrentes, « référentiellement vide» (Le Goffic 2020), mais réfère au contraire à un élément du contexte, antérieur le plus souvent :

*kuyumcuyan@unistra.fr 
a. Il râlait et se débattait contre la mort, mais ne pouvait prononcer une parole, ce que les médecins expliquèrent par la nature de ses blessures qui avaient traversé le poumon. Mérimée, Colomba

plus rarement postérieur :

b. Le lendemain on le réveilla à cinq heures; et, ce qui eût été cruel pour Mme de Rênal si elle l'eût su, à peine lui donna-t-il une pensée. Stendhal, Le Rouge et le Noir

ou dans lequel la proposition en ce + relatif est intercalée :

c. Je dois confesser, d'ailleurs, que lui et même le nommé Racine, ont fait chacun dans leur vie un vers assez bien rythmé, et qui a pour lui, ce qui est selon moi le mérite suprême, de ne signifier absolument rien. Proust, Du côté de chez Swann

Étant donné le trait sémantique attaché à ce, « désignateur des choses non nommées ou appréhendées comme telles » (Kleiber 1994 : 75), l'antécédent du pronom correspond à une entité «non classifiée », c'est-à-dire qui n'est pas, en ce point du discours où s'insère la relative, réduite à un nom. La proposition renvoie donc bien à un antécédent, en dehors du postiche $c e-$ ou à un antécédent auquel ce sert de relais devant le relatif -, mais cet antécédent n'est pas nominal ou équivalent à un groupe nominal, c'est un constituant d'une autre catégorie, groupe infinitival (GInf) ou même proposition (P) selon le cas, groupe dont l'identification contient d'ailleurs une marge d'imprécision, ainsi qu'on le verra. Or c'est bien la sélection idoine de l'antécédent qui conditionne, par-delà l'interprétation de l'expression anaphorique, celle de la relative introduite par son biais.

Faute de remplir cette condition de la coréférence avec un élément du contexte, ce qui/que, équivaut à un pronom indéfini et forme avec la relative la «nominalisation d'un actant exprimé sous forme d'une variable indéfinie » (Le Goffic 2020). Le «Groupe Pronominal» résultant ce $q u-P$ occupe alors dans la matrice une fonction nominale quelconque, par exemple sujet dans :

d. Ce qui nous empêche souvent de nous abandonner à un seul vice est que nous en avons plusieurs (La Rochefoucauld)

ce qui n'est jamais le cas des relatives neutres que nous nous proposons d'étudier. On peut le vérifier dans les trois exemples donnés ci-dessus où, quelle que soit sa position, la « relative» ne peut être décrite comme occupant une fonction syntaxique dans le reste de l'énoncé qu'on assimilerait ce faisant à une «matrice »-d'où sans doute l'hypothèse de l'« apposition» pour ces structures dans la GMF (815), hypothèse qui sauvegarde les apparences d'une «phrase complexe » à l'ensemble comprenant la relative. En conduisant cette hypothèse à son terme, on en viendrait d'ailleurs à soutenir avec $\mathrm{C}$. Touratier que :

[l']équivalence sémantique n'est pas due au pronom ce [...], mais au fait que le syntagme auquel [il appartient] est apposé à la proposition en question. Par conséquent, le ce qui introduit une apposition à une proposition n'est pas plus anaphorique [...] que le ce qui n'introduit pas une apposition à une proposition, et toutes les relatives introduites par ce ont, comme les relatives introduites par celui, un antécédent nominal dépourvu de contenu sémantique propre. (Touratier 1980 : 136).

Autrement dit, Touratier aligne le fonctionnement des « relatives anaphoriques » sur celui des non-anaphoriques en faisant porter le poids de la coréférence, qu'il ne nie nullement, à l'apposition, c'est-à-dire à un facteur syntaxique, au lieu de l'imputer directement à la sémantique. Il n'en demeure pas moins que le terme d'apposition appliqué à ces relatives 
neutres reste en l'espèce plutôt sous-déterminé, car comment une subordonnée peut-elle se trouver apposée à l'intégralité d'une matrice dont elle serait par ailleurs un constituant ? On est en réalité plus proche, avec de telles structures, de la coordination entre segments équivalents que de leur hiérarchisation par enchâssement :

un terme de $\mathrm{C}_{2}$ est prédestiné à devenir conjonction coordinative lorsqu'il renferme un représentant de $\mathrm{C}_{1}$ tout entier, soit explicitement (p. ex. « Il fait froid ; nous ne sortirons pas à cause de cela»), soit en cumul ou zéro (p. ex. " Je me suis levé, je suis sorti ensuite », c'est-à-dire « après cela »). On voit que dans ces cas le terme en question est un déterminant de tout ou partie de $\mathrm{C}_{2}$; mais le représentant qu'il contient a ce double effet de l'attirer, par une sorte de chiasme, vers le début de la phrase, et d'en faire le signe de son sujet psychologique. » (Bally 1965 : $§$ 94)

En transposant ces considérations aux relatives qui nous occupent, on avancerait avec $\mathrm{C}$. Muller :

[...] à la subordination externe, par rapport à un antécédent, se substituerait une subordination interne, par rapport au pronom relatif. Dès lors, il pourrait y avoir segmentation et un lien lâche entre le pronom relatif et son antécédent, ou même pas de lien du tout. La relative resterait alors une subordonnée parce que le verbe y serait dans la dépendance du pronom relatif. (Muller 1996 : 22)

Il n'y aurait alors pas plus de matrice que de subordonnée stricto sensu dans ces prédications à tiroir, dont l'une prend l'autre pour objet - exactement comme thème (Marchello-Nizia 1995 : 91 ; Glatigny 2003 : 243) - dans un développement « méta » à la récursivité potentiellement aussi duplicable - et aussi rarement réalisée pour les mêmes raisons - que l'enchâssement proprement dit. La proposition à relatif initial flotte pour ainsi dire tantôt à côté, tantôt au-dessus, de la prédication qu'elle prend pour objet. Comme elle s'appuie sur l'anaphore résomptive pour réaliser sa propre prédication, c'est même elle qu'il faudrait considérer comme "principale », en dépit de son marqueur d'enchâssement initial. Elle se trouve suspendue à l'autre proposition, certes, grâce à l'anaphore, mais sans y être intégrée au sens syntaxique du terme, c'est-à-dire en y occupant une fonction, aussi « périphérique » qu'on conçoive cette dernière. Elle semble juste destinée à réaliser une prédication au sujet de toute ou partie de la prédication dont elle se présente suivant le cas comme la suite - (a) - ou le commentaire - (b) et (c). En ce sens, elle est autonome et se suffit à elle-même si bien qu'on pourrait facilement lui substituer une phrase indépendante moyennant le remplacement d'une anaphore nominale au groupe initial $c e+$ relatif :

a'. Il râlait et se débattait contre la mort, mais ne pouvait prononcer une parole. Les médecins expliquèrent ce symptôme par la nature de ses blessures qui avaient traversé le poumon.

ou même une simple apposition :

b'. Le lendemain on le réveilla à cinq heures; et, comportement cruel pour Mme de Rênal si elle l'eût su, à peine lui donna-t-il une pensée.

c'. Je dois confesser, d'ailleurs, que lui et même le nommé Racine, ont fait chacun dans leur vie un vers assez bien rythmé, et qui a pour lui, mérite suprême selon moi, de ne signifier absolument rien.

même si la solution précédente reste possible :

b'. Le lendemain on le réveilla à cinq et à peine donna-t-il une pensée à Mme de Rênal. Cette légèreté eût été cruelle à la pauvre femme si elle en avait eu connaissance. 
c'. Je dois confesser, d'ailleurs, que lui et même le nommé Racine, ont fait chacun dans leur vie un vers assez bien rythmé, et qui a pour lui de ne signifier absolument rien. Cette propriété constitue selon moi le mérite suprême.

De telles substitutions sont inenvisageables dans les relatives à ce non anaphorique de type Ce qui nous empêche souvent de nous abandonner à un seul vice dans l'exemple d. cidessus. Le groupe commençant par ce $q u-P$ réalise alors un constituant essentiel de la phrase-hôte à la prédication de laquelle il est complètement intégré, au point qu'il ne peut ni s'en détacher, ni en former une autre à soi seul. À la "récursivité par auto-inclusion » qui caractérise ces « relatives périphrastiques », pour reprendre la désignation de la $G M F$, on opposera par conséquent une « récursivité linéaire» qu'illustrent nos prédications gigognes initiales (ex. a, b, c). On comparera en effet ce mode de prolifération des structures phrastiques à celle qu'A. Berrendonner caractérise de la façon suivante :

Elle [la récursivité [qui] ne tient pas à des homologies entre tout et partie] ne repose pas sur le fait qu'une structure macro-syntaxique puisse réapparaître à titre de constituant à l'intérieur d'elle-même, mais sur la simple répétition d'action ou de séquences d'actions. (Grammaire de la période 2012, (dorénavant GP) : 204)

En l'occurrence, le «rebond» qui se substitue à l'auto-inclusion s'opère grâce à l'anaphore, qui prend appui sur tout ou partie de la prédication précédente, la «met en boule » (Maillard 1972 : 93) pour en faire le support d'une nouvelle opération énonciative en chevillant les deux énonciations l'une à l'autre, non par le biais d'une circonstance ou d'un actant comme dans le mécanisme de la subordination « intégrative » tel que le conçoit P. Le Goffic (Grammaire de la Phrase française 1993 : § 24 ; cf. Muller 1996 : 68), mais par celui de la coréférence.

Conserver un cadre phrastique, fût-il complexe, pour décrire de telles structures, c'est probablement échouer à rendre compte du fonctionnement textuel - ou périodique dans le vocabulaire de la $G P$ - qui est leur raison d'être, et que G. Kleiber (à paraître) décrit grâce à la métaphore du «tuilage ». Ce qui invite par ailleurs à une approche transphrastique de ces relatives à antécédent neutre anaphorique, c'est qu'elles forment bien souvent à l'écrit, en français contemporain, des phrases graphiques autonomes. Aussi fragile que soit ce critère (GP : 3-19 ; Kleiber 2003), il atteste néanmoins de la perception d'une certaine « autonomie » de ces structures par les scripteurs eux-mêmes.

\subsection{En français préclassique}

L'ancien français connaissait lui aussi un « relatif neutre » devant lequel apparaît, à partir du $14^{\mathrm{e}}$ siècle selon Haase $(1975: \S 35)$, le pronom ce. Sa présence n'est pas systématique jusqu'au français classique, voire au-delà dans des emplois archaïsants, et le relatif neutre subsiste encore aujourd'hui dans des locutions figées telles que qui pis est, que je sache, et dans les incises de discours direct en français familier du type $q u$ ' $i(l)$ me dit/fait. Des relatifs neutres concernés - qui, que, dont et quoi-, seuls les deux premiers présentent une alternance significative entre formes avec et sans ce dans l'emploi anaphorique. Y a-t-il alors simple concurrence, c'est-à-dire variation libre, ou peut-on déceler des spécialisations dans les emplois? Ceux-ci correspondent-ils en outre à ceux du français moderne ou, hormis la fixation de ce devant le relatif, leur usage s'est-il modifié jusqu'à l'époque moderne ? Pour répondre à ces questions, il faut d'abord observer le fonctionnement de ces relatives dans les textes anciens, à l'instar de l'observation minutieuse entreprise par $\mathrm{M}$. Glatigny (2003) sur les relatifs dans les récits de voyage à l'époque préclassique. Nous nous proposons quant à nous d'étudier les constructions analogues à celles des exemples a, b, c dans un récit de fiction, L'Heptameron de Marguerite de Navarre (1559, 171000 mots ${ }^{1}$ ), et ce afin de déterminer plus précisément leur contexte d'apparition respectif avant toute investigation ultérieure. 


\section{Inventaire}

Dans L'Heptameron il n'y a aucune occurrence de ce dont avec un démonstratif anaphorique au sens défini sous 1.1, alors que les dont «neutres » abondent. Quoy, toujours prépositionnel, n'est lui non plus jamais précédé de ce dans les mêmes conditions. Qui et que sont quant à eux opposés : qui anaphorique neutre domine largement sur ce qui anaphorique (47 occurrences contre 13), tandis que c'est le contraire pour ce que (148 occurrences) par rapport à que (2 occurrences). Le tableau suivant montre quelle ponctuation précède ces relatives neutres :

Tableau 1 : ponctuation devant le relatif neutre

\begin{tabular}{|l|c|c|c|c|}
\hline & qui & ce qui & que & ce que \\
\hline virgule & 21 & 3 & 1 & 28 \\
\hline point-virgule & 9 & 4 & 1 & 71 \\
\hline deux points & 15 & 0 & 0 & 14 \\
\hline point & 2 & 6 & 0 & 35 \\
\hline Total & $\mathbf{4 7}$ & $\mathbf{1 3}$ & $\mathbf{2}$ & $\mathbf{1 4 8}$ \\
\hline
\end{tabular}

Étant donné le type de prédication qu'elles réalisent, ces propositions sont en effet nécessairement précédées d'un ponctuant, dont la «force » pourrait être en lien avec l'extension plus ou moins importante de l'antécédent. Néanmoins, ainsi qu'indiqué en introduction, la délimitation de ce dernier n'est pas toujours assurée. Il y a d'abord les cas où l'on peut hésiter entre relative neutre et relative à antécédent nominal :

1. Vous estes belle et jeune, vivant en toute compaignye bien joieusement; il n'y a nul en ceste court, qu'il ne voye la bonne chere que vous faictes au gentil homme dont vous avez soupson : qui fera juger chascun que s'il a faict ceste entreprinse, ce n'a esté sans quelque faulte de vostre costé.

L'antécédent de qui fera juger chascun... pourrait aussi bien être la bonne chere que vous faictes au gentil homme dont vous avez soupson, dont le groupe nominal (GN) la bonne chère est la "tête». Mais étant donné la prédication réalisée dans la relative, et la ponctuation qui la sépare des autres relatives construites en enfilade, son sujet représente plus probablement l'ensemble de la phrase précédente. On pourrait gloser ici qui par « cette situation», «tout cela». C'est la relative qui détermine finalement la sélection de son antécédent, rétroactivement, en guidant l'interprète vers le groupe le plus approprié à la prédication qu'elle contient (Kuyumcuyan 2011), avec des situations d'ambiguïté caractérisée encore plus nettes que dans l'exemple précédent :

2. Et là entendit que le Roy son frere s'en vouloit aller à Oly et Taffares; et, pensant que le voiage seroit long, entra en une grande tristesse, qui le mist jusques à deliberer d'essayer, avant partyr, si la saige dame luy portoit poinct meilleure volunté qu'elle n'en faisoit le semblant.

Quel est l'antécédent de qui ici? une grande tristesse qui le précède juste ou l'ensemble de la phrase? En d'autres termes, qu'est-ce qui déclenche la décision du gentilhomme d'essayer une dernière tentative ? De tels cas, qui ne sont pas très fréquents, n'ont pas été retenus dans le tableau précédent.

D'autre part, même quand l'antécédent n'est assurément pas un GN, on peut avoir un doute quant à sa délimitation exacte : est-il constitué de toute la proposition précédente, seulement d'une partie de celle-ci ou de toute la phrase?

3. lequel, incontinant qu'il fut à Thunis, ne faillit d'envoyer de ses nouvelles à ses amys, et, par homme fort seur, advertir Floride qu'il estoit en bonne santé et espoir de la reveoir ; qui fut à la pauvre dame le seul moyen de soustenir son ennuy. 
Dans un tel exemple, on admettrait aisément que seule la prédication contenue dans la complétive précédente - il estoit en bonne santé et espoir de la reveoir - est en définitive concernée par la prédication relative, mais rien n'interdirait non plus d'y inclure ce qui précède, qui participe également du soulagement de Floride, la pauvre dame. Dans de telles situations d'incertitude, c'est l'antécédent minimal, congruent avec la prédication relative, qui a systématiquement été retenu: subordonnée (P), groupe infinitif (GInf), groupe participial (Gpart)..., parce que cette délimitation a minima a paru plus précise préoccupation qui n'est d'ailleurs pas nécessairement celle du lecteur ancien ou moderne.

\subsection{Qui / ce qui}

Ces réserves signalées, voici comment se répartissent les antécédents pour (ce) qui :

Tableau 2 : Antécédents des relatifs neutres

\begin{tabular}{|c|c|c|c|c|c|}
\hline Relatif & Ponctuation & GInf & Gpart & P complétive & proposition maximale \\
\hline \multirow{4}{*}{ qui } &, & 8 & & 1 & 5 \\
\cline { 2 - 6 } & $;$ & 1 & & 1 & 7 \\
\cline { 2 - 6 } & $:$ & 3 & & 4 & 8 \\
\cline { 2 - 6 } & $\cdot$ & 0 & & 0 & 2 \\
\hline Total & & $\mathbf{1 2}$ & & $\mathbf{6}$ & $\mathbf{2 2}$ \\
\hline \multirow{3}{*}{ ce qui } & $;$ & 1 & & 2 & 0 \\
\cline { 2 - 6 } & $;$ & 2 & 1 & 1 & 1 \\
\cline { 2 - 6 } & $:$ & 0 & & 0 & 0 \\
\hline Total & & 0 & & 0 & $\mathbf{7}$ \\
\hline
\end{tabular}

Compte tenu du faible nombre d'occurrences, l'antécédent d'une relative neutre tend plutôt à être une proposition maximale (indépendante avec d'éventuelles subordonnées) après une ponctuation forte, et moins étendu (GInf ou PComp) après une ponctuation plus légère.

Le verbe de la relative constitue quant à lui un point essentiel pour l'analyse des enchaînements réalisés par ces tours, qu'on peut certes juger former des «collocations assez spécifiques et limitées »(Glatigny 2003 : 243-249), mais néanmoins significatives. En effet, dès qu'on met en relation l'antécédent situé dans P1 avec la prédication réalisée dans la relative, quelques constantes apparaissent, en fonction du verbe utilisé, au sujet des enchaînements textuels.

Tableau 3 : Verbes des relatives neutres en qui

\begin{tabular}{|c|c|c|c|c|c|c|}
\hline Relatif & ponctuant & être & $\begin{array}{c}\hat{e} \text { tre } \\
\text { cause/ } \\
\text { causer }\end{array}$ & $\begin{array}{c}\text { donner/être } \\
\text { occasion } \\
\text { donner envie }\end{array}$ & $\begin{array}{c}\text { tournure } \\
\text { factitive }\end{array}$ & $\begin{array}{c}\text { V. } \\
\text { plein }\end{array}$ \\
\hline \multirow{3}{*}{$q u i$} & $;$ & 8 & 3 & 1 & 1 & 1 \\
\cline { 2 - 7 } & $;$ & 1 & 1 & 3 & 4 & 0 \\
\cline { 2 - 7 } & $:$ & 4 & 3 & 1 & 5 & 2 \\
\hline Total & & 1 & 0 & 0 & $\mathbf{1 1}$ & $\mathbf{3}$ \\
\hline
\end{tabular}

\subsection{1 Être}

Avec un antécédent GInf, le verbe être à l'imparfait, attributif, exprime une évaluation propre à un ou plusieurs personnages de l'histoire, dans un développement d'arrière-plan caractéristique d'un « effet point de vue » narratif : 
4. Le pauvre gentilz homme, voyant le secours que ces deux luy avoient faict, après les avoir embrassé et remercié les pria de ne les habandonner poinct, qui leur estoit requeste fort aisée.

L'aspect global du passé simple, avec le même verbe être, et un antécédent infinitival ou propositionnel, entérine la réalisation du procès évoqué dans l'antécédent en en précisant une circonstance de manière, très souvent grâce à la périphrase (ce) qui ne fut pas sans GN / VInf - « non sans $\mathrm{X}$ »-, selon le mécanisme du «complément différé » (BlancheBenveniste $1997: 114)$ :

5. Et, en ce disant, luy monstra l'anneau qu'elle avoit au doigt comme le signe de sa parfaicte amityé, qui ne fut sans grandes larmes:

6. Mais cela ne les garda d'estre prins et mis prisonniers, qui ne fut sans estre mocquez et huez d'hommes et femmes.

Au présent, la relative avec être exprime plutôt une loi générale directement prise en charge par le narrateur dans la situation d'interlocution propre au récit oral représenté dans l'ouvrage, que l'antécédent soit infinitival ou propositionnel :

7. Sans poinct de faulte, dist Ennasuitte, ce debvoit estre quelque glorieuse folle, qui, par sa resverie des Cordeliers, pensoit estre si saincte qu'elle estoit impecable, comme plusieurs d'entre eulx veullent persuader à croyre que par nous-mesmes le povons estre, qui est ung erreur trop grand.

8. Ce n'est pas mon intention, dist Nomerfide, car je parlerois contre l'experience que j'ay des femmes, mais je entends que ung plaisir non accoustumé, comme d'espouser l'homme du monde que l'on ayme le mieulx, doibt estre plus grand que de le perdre par mort, qui est chose commune.

\subsubsection{Les tours causatifs}

L'ensemble constitué par être... cause, causer, contraindre, donner occasion / envie ainsi que les tours causatifs proprement dits (périphrase avec faire + VInf) servent quant à eux à souligner les enchaînements causaux sur lesquels repose censément la trame narrative. Non sans une certaine «lourdeur» pour le lecteur contemporain, le procès de la relative se trouve de la sorte arrimé à celui qui précède au moyen du pivot pronominal :

9. A l'heure, pensa le prince que le seigneur des Cheriotz estoit en la chambre de sa dame, et que, pour craincte de luy, n'osoit descendre ; qui le feit encores promener longtemps.

10. Et, pour en divertir sa fantaysie, s'esloingna quelques jours de sa veue : qui luy causa une melencolye si grande, que les medecins lui ordonnerent la saignée.

Le procès ou l'état impliqué par l'antécédent - celui qu'exprime le verbe à l'infinitif après faire, ou le GN après causer - est en ces cas aussi bien une action (9), qu'un état mental ou psychologique (10). Le passé simple confère alors à la relative sa pleine valeur « continuative», ce qui n'était pas aussi nettement le cas dans les exemples 5 et 6 cidessus. La relation de cause à effet est par ailleurs souvent préparée par un tour corrélatif intensif dans l'antécédent :

11. Et, pour ce que ses maistre et maistresse l'aymoient autant que homme de son estat qui fust en leur maison, ne pouvoit si souvent aller veoir sa femme qu'il eust bien voulu : qui fut occasion dont elle oblya tellement son honneur et conscience, qu'elle alla aymer ung jeune homme, 
L'explicitation du lien causal au moyen du groupe verbal est plus fréquente que l'inverse, à savoir la construction du verbe «plein » directement après le relatif, dont on ne trouve que trois exemples au total avec qui :

12. Ainsy qu'il commencea à se chaulfer en mangeant, son pain de sucre commencea aussy à desgeller, qui remplit toute la chambre de telle senteur que le pain estoit.

13. Le beau pere, après luy avoir dict plusieurs propos de la grande amityé qu'il luy portoit, luy voulut mectre la main au tetin: qui fut par elle repoulsé comme elle debvoit; $^{2}$

14. Si eurent-ils si habilement cueilly leurs prunes, pour ce qu'elles estoient meures, que Olivier mesme ne le pouvoit croire, n'eust esté qu'il veid la fille tirant la veuë contre bas, et monstrant visaige honteux : qui luy donna marque de la verité,

\subsubsection{Spécificité de ce qui ?}

Les verbes des relatives avec ce qui se distinguent partiellement des précédents :

Tableau 4 : Verbes des relatives neutres en ce qui

\begin{tabular}{|c|c|c|c|c|}
\hline & & être & $\begin{array}{l}\text { être fait/ } \\
\text { exécuté }\end{array}$ & V plein \\
\hline \multirow{3}{*}{ ce qui } &, & 0 & 1 & 2 \\
\cline { 2 - 5 } & $;$ & 1 & 3 & 0 \\
\cline { 2 - 5 } Total & & 0 & 2 & 4 \\
\hline
\end{tabular}

Une seule occurrence de être attributif trouvée dans le corpus, à l'imparfait, tout à fait identique à celles signalées avec qui (2.1.1) :

15. car son intention estoit de l'avoir pour femme ; ce qui estoit chose fort raisonnable, car il estoit beau, riche et de bonne maison.

On relève à deux reprises dans la dernière colonne des verbes d'appréciation, plaire et déplaire respectivement, qui prennent pour thème la situation précédente en la soumettant à l'appréciation d'un nouveau personnage, commode moyen d'effectuer une transition thématique d'une séquence à thème constant à la suivante :

16. Entre aultres, il y avoit ung jeune gentil homme, nommé le seigneur des Cheriotz, qui la poursuivoit de si près, qu'il ne falloit d'estre à son habiller et son deshabiller, et tout le long du jour, tant qu'il povoit estre auprès d'elle. Ce qui ne pleut pas au prince de Belhoste, pource qu'il luy sembloit que ung homme de si pauvre lieu et de si mauvaise grace ne meritoit poinct avoir si honneste et gratieux recueil : dont souvent il fasoit des remonstrances à ceste dame.

Mais le plus remarquable est la fréquence de diathèses passives dans les relatives en ce qui - 7 sur 13 -, non moins que leur caractère stéréotypé : ce qui fut fait / exécuté / eut esté faict. Tout aussi significatif est leur antécédent: systématiquement une séquence de discours représenté, selon le cas direct :

17. «[...] Parquoy allez-vous-en et mectez le curé en prison. Je suis seur qu'il confessera la verité. » Ce qui fut faict selon son commandement, non sans grandes remontrances pour le scandalle qu'ilz faisoient à cest homme de bien.

ou indirect : 
18. Le rapport en fut faict à la Royne, et quelcun de la compaignie s'advisa qu'il estoit bon de regarder derriere la porte auprès de laquelle on l'avoit prins ; ce qui fut faict et trouva l'on ce que l'on cerchoit :

En somme, ce qui fut fait articule le passage d'une requête à son exécution en escamotant l'agent de celle-ci, mais en mettant au premier plan la "réussite » de l'acte illocutoire initial. L'anaphore résomptive intègre ce faisant la séquence énonciativement hétérogène dans le cours narratif en la traitant comme un événement quelconque, susceptible comme tel d'en entraîner d'autres. On note la présence de ce dans cette insertion de discours représenté (DR), rôle qu'il joue par ailleurs dans les incises de discours direct dans l'ancienne langue et encore parfois au $16^{\mathrm{e}}$ siècle. On retrouve en une autre occurrence à la fois ce, le passif, et du DR, mais dans un acte illocutoire d'information cette fois. Cependant, la négation du verbe de la relative montre que l'acte en question n'a pas été couronné de succès, en ce sens qu'il n'a pas réussi à modifier la représentation de son destinataire comme le locuteur en avait manifestement l'intention :

19. Et vint dire au seigneur de Jossebelin, que le gentil homme, auquel il se fyoit tant, alloit trop souvent en la chambre de sa soeur, et aux heures où les hommes ne doibvent entrer. Ce qui ne fut creu pour la premiere foys, de la fiance qu'il avoit à sa seur et au gentil homme.

Il ressort de ces différents emplois que la relative neutre relève d'une stratégie textuelle qu'on pourrait qualifier d'explicite, en ce que l'articulation des séquences y est manifeste, et tend même à être surmarquée, en particulier aux plans informationnel et (chrono)logique. Le relatif neutre y devient alors un marqueur d'intégration qui sert tout à la fois à séparer et recomposer les différentes étapes d'un schéma praxéologique. Outil de cohérence emprunté à la syntaxe intraphrastique, il est devenu un simple ligateur textuel transphrastique, devant lequel ce, encore rare dans notre texte narratif, réfère essentiellement à du discours représenté. Nulle part ce rôle de ligateur n'apparaît mieux qu'avec les périphrases causatives, les tournures factitives, le passif ou même les verbes «pleins » dans la relative, où la présupposition entre propositions successives reste le principe organisateur de la progression textuelle (Bonnard 1978). Même le verbe être s'inscrit dans ce cadre, pour certains de ses emplois tout au moins, y compris la périphrase circonstancielle différée qui ne fut sans $X$. Il en résulte que les emplois proprement attributifs de être sont assez rares, plus fréquents au présent qu'au passé simple, contextes dans lesquels la relative est généralement remplaçable par une simple apposition. La tournure joue alors véritablement le rôle d'une "proposition incidente », qui pourrait d'ailleurs être intégrée ailleurs dans la phrase. Tel n'est pas le cas des autres relatives dont on vient de souligner l'apport essentiel dans la progression, et qu'on pourrait pour cette raison qualifier de relatives neutres continuatives, lesquelles s'opposeraient donc aux incidentes ou parenthétiques dont il vient d'être question.

\subsection{Que / ce que}

Il est impossible, pour que, de comparer les emplois avec et sans ce en raison du très faible nombre d'occurrences trouvées sans ce (cf. tableau 1). Des doutes sur l'antécédent, analogues à ceux qu'on rencontrait avec qui neutre, subsistent néanmoins dans quelques rares cas :

20. Laquelle, comme j'ay dict, bailla par la grille à son frere tout le discours de sa piteuse histoire. Ce que le frere porta à sa mere; laquelle, toute desesperée, vint à Paris, où elle trouva la Royne de Navarre, seur unicque du Roy, à qui elle monstra ce piteux discours, en luy disant : 
Le «discours de la piteuse histoire » dont il est ici question, c'est le texte écrit qu'une religieuse transmet par la grille d'un couvent à son frère, et que celui-ci «porte » à sa mère : l'antécédent de ce que est bien le GN antécédent, pourquoi un relatif neutre en ce cas? De même :

21. [...] et luy confessa l'amityé d'eulx deux, ce que Floride avoit tousjours celé, la priant luy vouloir ayder d'avoir souvent de ses nouvelles, et de retirer bien tost Floride avecq elle.

Ici également, ce que cache Floride, c'est l'amityé d'eulx deux, GN qui ne requiert pas l'emploi du relatif neutre. Tandis que la forme qui, en se prêtant aux deux lectures neutre et non neutre, favorise ce type d'ambiguïté (cf. supra : 2), on pourrait penser que ce que, du fait de sa distinction d'avec que, y échappe. Tel n'est donc pas toujours le cas, même si les exceptions sont rares.

Tableau 5 : Catégories d'antécédents et de verbes des relatives en ce que

\begin{tabular}{|c|c|c|c|c|c|c|c|}
\hline \multicolumn{4}{|c|}{ ANTECEDENT } & \multicolumn{4}{|c|}{ VERBE DE LA RELATIVE } \\
\hline ponctuant & catégorie & total & $\begin{array}{l}\supset \\
\mathrm{DR}\end{array}$ & faire & « subjectif » & parole & événement \\
\hline \multirow{4}{*}{. } & complétive & 8 & 6 & 4 & 2 & 2 & 0 \\
\hline & Relative & 2 & 0 & 0 & 2 & 0 & 1 \\
\hline & GInf & 21 & 13 & 14 & 3 & 2 & 2 \\
\hline & $\mathrm{P} \max$. & 5 & 2 & 0 & 3 & 2 & 0 \\
\hline \multirow{4}{*}{; } & complétive & 22 & 11 & 8 & 1 & 12 & 1 \\
\hline & Relative & 2 & 0 & 1 & 0 & 1 & 0 \\
\hline & GInf & 38 & 26 & 22 & 1 & 7 & 4 \\
\hline & $\mathrm{P} \max$. & 4 & 1 & 0 & 0 & 2 & 2 \\
\hline \multirow{4}{*}{ : } & complétive & 4 & 3 & 1 & 0 & 2 & 1 \\
\hline & Relative & 1 & 1 & 0 & 0 & 0 & 1 \\
\hline & GInf & 7 & 6 & 3 & 3 & 2 & 2 \\
\hline & $\mathrm{P} \max$. & 1 & 1 & 0 & 1 & 0 & 0 \\
\hline \multirow{4}{*}{, } & complétive & 7 & 3 & 2 & 3 & 0 & 2 \\
\hline & Relative & 1 & 0 & 0 & 1 & 0 & 0 \\
\hline & GInf & 12 & 8 & 7 & 1 & 2 & 2 \\
\hline & $\mathrm{P} \max$. & 2 & 0 & 1 & 0 & 0 & 1 \\
\hline
\end{tabular}

\subsubsection{L'antécédent de ce que}

Il ressort du tableau 5 que, quelle que soit la ponctuation précédant ce que neutre, son antécédent le plus fréquent reste le GInf - la moitié ou plus du total -, suivi de la complétive au sens large (incluant les «percontatives») - environ le quart. Très loin derrière arrivent la proposition maximale et enfin la relative, soit la formule suivante pour quantifier les catégories d'antécédent :

\section{GInf $>$ Compl. $>$ Prop. maxi. $\geq$ Relative}

Les chiffres du tableau sont à prendre avec précaution, c'est pourquoi ils ne sont pas assortis de pourcentages qui pourraient induire en erreur. Un ce que anaphorique neutre et un ce que indéfini (cf. supra 1.1) étant en effet identiques pour un traitement de texte, chaque occurrence est à trier "manuellement», et les oublis sont toujours possibles, sans compter les ambiguïtés déjà signalées. Il s'agit simplement ici d'indiquer des ordres de grandeur, ce qui suffit au caractère de la présente observation, qui essaye de décrire ponctuellement un phénomène, non de le chiffrer précisément, car cela réclamera d'autres outils et un autre cadre. 


\subsubsection{La relative avec faire}

C'est le verbe faire qui est de loin le plus fréquent dans la relative. Employé comme vicaire, il reprend, en association avec le relatif, le procès exprimé dans son antécédent équivalent ainsi à la reprise par le faire aussi (Delaveau 2001 : 142 ; cf. Combettes 2003 : 196) -, c'est-à-dire le verbe à l'infinitif quand il s'agit d'un GInf :

22. Sa seur l'envoya querir et luy feit fort bonne chere, la priant de la venir souvent veoir ; ce qu'elle faisoit quant il y avoit quelques nopces ou assemblée,

ou le verbe conjugué d'une complétive :

23. Et, pource qu'il estoit de fort bonne heure, le marié ne voulut laisser la dance, mais y estoit tant affectionné, qu'il sembloit qu'il eut oblyé sa femme ; ce que n'avoit pas faict le Cordelier,

Le contexte le plus fréquent de reprise d'un verbe à l'infinitif est celui du premier exemple : le GInf est l'objet d'un verbum dicendi / d'un verbe d'attitude propositionnelle dans une situation de récit de paroles / de pensées, le procès à l'infinitif y est donc envisagé sur un mode virtuel, dont la relative neutre indique aussitôt au minimum la réalisation :

24. Toutesfois, pensa en elle-mesme qu'elle cercheroit quelque autre moyen dont la Royne ne sçauroit rien; ce qu'elle feyt.

en l'assortissant éventuellement de circonstances diverses :

25. Le mary, voyant cela, commanda à sa femme de le baiser ; ce qu'elle feit, sans faire semblant de vouloir ne non voulloir ce que son mary luy commandoit.

On note en ce cas le passé simple du verbe faire, dont l'aspect global est essentiel à l'effet de bouclage induit par la relative. On pourrait parler ici de micro-séquences praxéologiques dans lesquelles l'antécédent ouvre un programme d'action que conclut la relative neutre au passé simple, selon un séquençage progressif où chaque étape du parcours est soigneusement balisée, dans une progression pas à pas qui ne laisse nulle place à l'ellipse narrative, et encore moins au raccourci que réalisaient dans les mêmes conditions les relatives de type qui ne fut sans (2.1.1):

26. Vespres oyes, allerent souper, qui ne fut tout le soir sans parler des comptes qu'ils avoient oyz, et sans chercher par tous les endroictz de leurs memoires, pour veoir s'ilz pourroyent faire la Journée ensuyvante aussi plaisante que la premiere.

Là où l'exemple avec qui téléscope le procès du déplacement et celui du repas dont il précise une circonstance de manière en anaphorisant le verbe à l'infinitif de la périphrase verbale temporelle, les relatives en ce que fit $x$ déploient l'intégralité du parcours effectué en explicitant l'actualisation du procès à l'infinitif par la reprise en faire au passé simple.

\subsubsection{Les relatives à verbe de parole}

Le deuxième groupe de verbes bien représentés dans la relative neutre est constitué de verbes de parole au sens large. On relève d'une part dire, mander, remontrer, déclarer, ainsi que la périphrase faire entendre, plutôt réservés au transfert de propos représentés antérieurement :

27. Au bout de quelque temps qu'ilz vivoient tous deux comme ilz avoient accoustumé, le gentil homme maryé rentra en soupson plus que jamais et commanda à sa femme qu'elle ne lui feit plus le visaige qu'elle lui faisoit ; ce qu'elle dist au compaignon de son mary, le priant de luy-mesmes se vouloir abstenir de parler plus à elle, car elle avoit commandement d'en faire autant de luy. 
28. car il luy avoit commandé de faire, en toutes ses choses, hors mys une, aussi bonne chere à son compaignon comme à luy, et neanmoins luy defendoit parler à luy, si elle n'estoit en grande compaignye. Ce qu'elle feit entendre au compaignon de son mary,

d'autre part des verbes décrivant les actes illocutoires réactifs de personnages engagés dans une activité conversationnelle, typiquement la ratification - l'accord ou le refus consécutif à une requête formulée juste auparavant par l'interlocuteur :

29. Et, quant il veid qu'il estoit contrainct d'obeyr, demanda congé à la marquise de dire adieu à Poline, et puis, que jamais il ne parleroit à elle ; ce que luy fut accordé,

30. Le seigneur d'Avannes, crainctif, ayant la larme à l'oeil, la suplia très fort, que, pour seureté de ses parolles, elle le voulsist baiser ; ce qu'elle refusa, luy disant que pour luy elle ne romproit poinct la coustume du pays.

voire simplement le vœu d'un personnage :

31. et, un beau dimanche que tout le monde dansoit publiquement, un escholier se voulut mettre en dance, ce que ne permirent lesdistz marroufles. Pantagruel

mais aussi toutes sortes de promesses elles aussi consécutives aux propos précédents :

32. Et monstroit bien qu'elle ne prenoit point desplaisir, quant il parloit à elle, en luy disant souvent qu'il ne tinst plus de telz propos ; ce qu'il luy promist, pour ne perdre l'aise et honneur qu'il avoit de l'entretenir.

On a compté au nombre de ces « verbes de parole » : jurer, promettre, accorder, octroyer.

\subsubsection{Les verbes « subjectifs"}

On a regroupé sous cette étiquette les verbes impliquant la présence d'un sujet énonciateur : verbes d'attitude propositionnelle (croire, trouver, savoir, penser), verbes de sentiment (porter ou supporter, craindre, espérer, endurer) et de perception (regarder, adviser, ö̈r, voir). Dans la relative neutre, de tels verbes dont l'antécédent, via la reprise en ce que, est l'objet, opèrent un changement de point de vue narratif en déplaçant le centre de perspective sur un personnage jusque-là en point de vue externe :

33. Mais il la retint entre ses bras, et, par bonnes et doulces parolles, l'asseurant de l'aymer plus que celluy qui l'aymoit et de celler ce qui touchoit son honneur, si bien qu'elle n'en auroit jamais blasme. Ce que la pauvre sotte creut ;

ou sur un personnage non encore mentionné dans le récit :

34. et ung matin, avant que personne fut esveillé en sa maison, la mena toute en chemise faire le crucifix sur la neige, et, en se jouant tous deux à se bailler de la neige l'un l'aultre, n'oblierent le jeu des Innocents. Ce que advisa une de leurs voisines, qui s'estoit mise à la fenestre qui regardoit tout droict sur le jardin, pour veoir quel temps il faisoit ;

ou sur un personnage présent jusque-là seulement en arrière-plan :

35. Le gentil homme, qui ne se donnoit de garde, estant seur que sa femme ne les povoit veoir, baisa sa chamberiere : ce que pour une foys sa femme endura sans dire mot,

Lorsque le personnage reste le même, ce que parenthétise sa représentation précédente pour en faire l'objet d'une nouvelle appréciation dans une opération de « métaprédication », si bien qu'on peut tout de même a minima parler en ce cas d'un changement d'énonciateur de « point de vue » au sens ordinaire du terme (Ducrot 1984) : 
36. Et, quant il fut retourné en son prieuré, il ne povoit trouver repos: parquoy en grande inquietude passoit les jours et les nuictz en cerchant les moyens comme il pourroit parvenir à son desir, et faire d'elle comme il avoit faict de plusieurs autres. Ce qu'il craingnoit estre difficille pource qu'il la trouvoit saige en parolles, et d'un esperit si subtil, qu'il ne povoit avoir grande esperance, et, d'autre part, se voyait si laid et si vieulx, qu'il delibera de ne luy en parler poinct, mais de chercher à la gaingner par craincte.

\subsubsection{Relatives neutres en ce que et progression informationnelle}

Le «chevillage» réalisé par la relative en ce que, étant donné la référence résomptive d'une part, la fonction du relatif de l'autre, s'articule aux prédicats verbaux des deux propositions concernées. Mais le sémantisme du verbe de la relative influe également sur le caractère de la progression réalisée. On pourrait en représenter les principales variantes évoquées ci-dessus par le tableau suivant :

Tableau 6 : Verbe de la relative neutre et progression informationnelle/narrative

\begin{tabular}{|c|c|c|c|c|}
\hline & Antécédent & \multicolumn{3}{|c|}{ Relative neutre } \\
\hline $\mathrm{V}$ relative & & thème & rhème & enchaînement \\
\hline \multirow{3}{*}{ faire } & \multirow{3}{*}{$\begin{array}{l}\text { Prédicat virtuel } \mathrm{P} \\
\text { Sujet / contrôleur } \mathrm{S}_{1}\end{array}$} & \multirow{3}{*}{$\begin{array}{l}\text { ce que } \\
=\mathrm{P}\end{array}$} & $\begin{array}{l}1 \% \text { fait/fit } S_{1} \\
\pm \\
\left\{\begin{array}{l}\mathrm{GP} \\
\text { adv-ment } \\
\text { quand } Q\end{array}\right.\end{array}$ & $\begin{array}{l}\text { actualisation P } \\
\text { circonstance } \\
\text { de } \\
\text { l'actualisation }\end{array}$ \\
\hline & & & $2 \%$ (ne) fait(pas) $S_{2}$ & $\begin{array}{l}\text { changement pers. } \\
=\text { transition thématique }\end{array}$ \\
\hline & & & $\begin{array}{l}3 \% \text { fit } S_{1} \\
\text { changement de } \\
\text { tiroir verbal }\end{array}$ & $\begin{array}{l}\text { actualisation « nue » } \\
\text { de } \mathrm{P}= \\
\text { progression chronologique }\end{array}$ \\
\hline \multirow{2}{*}{$\begin{array}{l}\text { Verbe } \\
\text { de } \\
\text { parole }\end{array}$} & $\begin{array}{c}-S_{1} \text { procès de parole } S_{2} \\
=P\end{array}$ & $\begin{array}{l}\text { ce que } \\
=P\end{array}$ & dit $S_{2} \grave{a} S_{3}$ & $\begin{array}{l}\text { rapport interne: } \\
\text { nouveau destinataire }\end{array}$ \\
\hline & $-\mathrm{S}_{1}$ demande $x$ à $\mathrm{S}_{2}$ & $\begin{array}{l}\text { ce que } \\
=x\end{array}$ & $S_{2}\left\{\begin{array}{l}\text { accorde } \\
\text { refuse } \\
\text { promet }\end{array}\right.$ à $S_{1}$ & $\begin{array}{l}\text { enchaînement } \\
\text { illocutoire } \\
(\rightarrow \text { dialogue })\end{array}$ \\
\hline $\begin{array}{c}\text { Verbe } \\
\text { subjectif }\end{array}$ & $\begin{array}{l}\text { Attitude Propositionnelle } \\
\text { Perception / Sentiment } \\
\qquad \mathrm{S}_{1} \mathrm{P} \\
\end{array}$ & $\begin{array}{l}\text { ce que } \\
=\mathrm{P}\end{array}$ & $\begin{array}{l}\quad \mathrm{S}_{1}{ }_{1} / \mathrm{S}_{2} \\
1 / \mathrm{VAPS} \mathrm{S}_{1} / \mathrm{S}_{2} \\
2 / \mathrm{VPS}{ }_{1} / \mathrm{S}_{2} \\
3 / \mathrm{VS} \mathrm{S}{ }_{1} / \mathrm{S}_{2} \\
\end{array}$ & $\begin{array}{l}\text { changement } \\
\text { de point de vue } \\
\text { et/ou de personnage }\end{array}$ \\
\hline
\end{tabular}

\section{Conclusion : un marqueur polyvalent}

Prise au pied de la lettre et sans se référer au latin où la situation est sans doute plus complexe qu'on ne le présente généralement (Touratier 1980:450-452), l'expression de « relatif de liaison» décrit assez bien le rôle du relatif neutre, comme le relève aussi J.-P. Seguin :

Cette appellation [relatifs de liaison], qui sent sa grammaire latine, rappelle l'espèce de contradiction qui existe entre un morphème subordonnant (donc pour nous intraphrastique) et l'enchaînement marqué à la place précise du point, qui ainsi n'a pas totalement sa fonction séparatrice. Par rapport à nos habitudes, on pourrait y voir le germe de l'hyperbate [...]. Est-ce l'indice d'une instabilité du rôle du point, ce qui serait pour nous une transgression de la frontière ponctuelle? Si cela nous paraît, à nous modernes, un élément sensible, c'est que nous avons 
depuis établi une ligne de partage entre coordination et subordination d'une part, entre continuité formelle et rupture formelle d'autre part. (Seguin $2003: 86$ )

Grâce à l'insertion d'un fragment plus ou moins étendu d'une première proposition dans celle qui la suit, à titre de sujet ou de partie du prédicat, celui-ci « cheville» les deux séquences phrastiques l'une à l'autre sans les hiérarchiser pour autant, assurant ainsi autant la continuité que la progression informationnelle, d'où le terme de "pronom conjonctif » naguère proposé par G. Antoine (1958 : 326). Le petit nombre de verbes qui entrent le plus fréquemment dans ces "phrases » relatives montre néanmoins le caractère quelque peu mécanique de ces enchaînements, qui semblent réservés, dans le corpus restreint auquel l'observation présente s'est limitée, à certaines configurations textuelles bien particulières : l'implication avec les tours causatifs (neutre qui); les relations illocutoires réactives dans un contexte de DR avec ce qui et faire au passif d'une part, ce que et un "verbe de parole » (au sens défini en 2.2.3) de l'autre ; l'actualisation d'un programme praxéologique énoncé d'abord sous forme virtuelle (ce que et faire vicaire) ; le changement de "point de vue » narratif enfin (changement de personnage ou d'énonciateur) lorsqu'intervient un verbe «subjectif» (au sens indiqué sous 2.2.4) dans une relative en ce que. De tels enchaînements « linéaires » - au sens informationnel du terme - ont pour caractéristique de contribuer au développement «horizontal» du récit, sa «progression syntagmatique » si l'on veut, laquelle greffe à chaque événement - action proprement dite, propos, projet celui qui est censé en découler dans la trame narrative. C'est pourquoi on pourrait rapprocher les tours correspondants des relatives "continuatives » à antécédent nominal (Combettes 1992: 124-126) : le type d'antécédent excepté, elles servent de la même manière la progression narrative.

A ces relatives continuatives s'opposent, dans L'Heptaméron tout au moins, les relatives qui pourraient a contrario être qualifiées de «métaprédicatives » : parenthétiques ou incidentes, leur rôle consiste à renchérir sur la prédication initiale plutôt qu'à la prolonger, que ce soit par un commentaire - interne autant qu'externe - ou par un complément détaché d'étendue variable. C'est une dimension «verticale» - ou «paradigmatique »- du texte qui s'en trouve cette fois développée, au moyen, toujours dans le corpus considéré, de relatives introduites par qui et dont le verbe est être, principalement. Ce sont ces relatives-là qui perdurent selon Haase, même sans démonstratif, au siècle suivant ( $\$ 35)$, tandis que les continuatives neutres, avec ou sans ce, sans disparaître tout à fait de la littérature narrative classique (Glatigny 2003 : 249-255), y sont cependant bien moins répandues. On n'en trouve par exemple plus trace un siècle plus tard dans La Princesse de Clèves (1678), alors qu'y subsistent quelques relatives parenthétiques. Surtout, les relatives neutres n'y présentent plus ce caractère de routine qu'elles ont dans le récit préclassique, dans la mesure où le rôle de ligateur du relatif s'est alors pour l'essentiel trouvé transféré à la conjonction (Siouffi 2003: 62-63). Le « connecteur » a momentanément triomphé de l'anaphore, avant que celle-ci ne revienne en force, dans les récits (dès le $18^{\mathrm{e}}$ selon un rapide sondage), mais aussi dans d'autres genres moins explorés comme les textes de presse du français contemporain, où l'on peut assimiler le rôle de des relatives neutres à celui d'un connecteur (Kuyumcuyan 2011). On remarquera d'ailleurs au passage que nos trois exemples modernes initiaux illustrent respectivement les types continuatif (a) et parenthétique (b et c). Raison de plus pour inviter à une investigation plus poussée de ces outils d'enchaînement textuel qui empruntent tantôt à l'anaphore et tantôt au connecteur (Berrendonner 1983), et à poser les jalons pour leur observation systématique en diachronie, ainsi que l'avait déjà entrepris G. Antoine (1958) en son temps.

\section{Références bibliographiques}

Antoine, G. (1958). La coordination en français. Paris : d'Artrey. 
Bally, C. (1965). Linguistique générale et linguistique française. Berne : Francke. $4^{\mathrm{ème}}$ édition.

Berrendonner, A. (1983). Connecteurs pragmatiques et anaphore. Cahiers de Linguistique Française $5,215-246$.

Berrendonner, A. et al. (2012). Grammaire de la période. Berne : P. Lang. Abrégé en GP

Blanche-Benveniste, C. et al. (1997). Approches de la langue parlée en français. Gap-Paris : Ophrys.

Bonnard, H. (1971-1978). Entrée Coordination du Grand Larousse de la langue française.

Combettes, B. (1992). L'organisation du texte. Metz: Centre d'Analyse Syntaxique de l'Université de Metz.

Combettes, B. (2003). Variation dans la structure du syntagme verbal. Évolution et variation en français préclassique. B. Combettes éditeur. Paris : Champion, 171-210.

Delaveau, A. (2001). Syntaxe. La phrase et la subordination. Paris : A. Colin.

Ducrot, O. (1984). Le dire et le dit. Paris : Minuit.

Glatigny, M. (2003). Les relatifs. Évolution et variation en français préclassique. B. Combettes éditeur. Paris : Champion, ; 211-270

Haase, A. (1898). Syntaxe française du XVII siècle. Paris, Picard et fils. Nouvelle édition traduite et remaniée par M. Obert, rééd. 1975, Paris : Delagrave.

Kleiber, G. (1994). Anaphores et pronoms, Louvain-la-Neuve : Duculot.

Kleiber, G. (2003). Faut-il dire adieu à la phrase ? L'Information grammaticale 98, 17-22.

Kleiber, G. (2020). Ce faisant / Ce disant : comment fonctionnent-ils ?.Langue française 205, 65-80

Kuyumcuyan, A. (2011). Syntaxe et sémantique du relatif de liaison en français moderne. Saillance, Aspects linguistiques et communicatifs de la mise en évidence dans un texte. Dirigé par $\mathrm{O}$. Inkova. Annales littéraires de l'Université de Franche-Comté, ${ }^{\circ}{ }^{\circ}$ 897. Série Recherches en linguistique. Presses universitaires de Franche-Comté. 187-204.

Le Goffic, P. (1993). Grammaire de la Phrase française. Paris : Hachette.

Le Goffic P. (2020). Ce qui, ce que : essai de description syntaxique et sémantique. Langue française 205, 81-99.

Maillard, M. (1972). Anaphores et cataphores. Communications 19, 93-104

Marchello-Nizia, C. (1995). L'évolution de français. Ordre des mots, démonstratifs, accent tonique. Paris : A. Colin.

Muller, C. (1996). La subordination en français. Paris : A. Colin.

Riegel, M., Pellat, J.-C. \& Rioul, R. (2009). Grammaire méthodique du français. Paris, PUF, $4{ }^{\mathrm{ème}}$ éd. entièrement revue. Abrégé en $G M F$.

Seguin, J.-P. (2003). Enchaînement et usage du point. In Evolution et variation en français préclassique. B. Combettes éditeur. Paris. Champion. 69-137.

Siouffi, G. (2003). Le regard des grammairiens. In Evolution et variation en français préclassique. B. Combettes éditeur. Paris. Champion. 19-68.

Touratier, C. (1980). La relative, essai de théorie syntaxique. Paris : Klincksieck.

\footnotetext{
${ }^{1}$ Nous avons travaillé sur [L']heptaméron [Document électronique] / Marguerite de Navarre; [texte établi sur les ms. par Michel François, et en tous points identique à l'édition à l'édition des classiques Garnier réédité en 2005 à Paris] disponible sur le site de la bnf, enregistré le 05/01/17, qui présente l'inconvénient d'être dépourvu de pagination.

${ }^{2}$ Comme le signale un relecteur anonyme, il se pourrait néanmoins fort bien que l'antécédent de qui soit ici le beau pere (éloignement tout à fait courant à l'époque), d'autant que le verbe repousser était alors plutôt compatible avec un objet animé (repousser un ennemi), auquel cas on se trouverait devant un relatif ordinaire à antécédent nominal.
} 